Nature Geosciences

December 2016, Volume 9 Issue 12 Pages 857-858

http://dx.doi.org/10.1038/ngeo2850

http://archimer.ifremer.fr/doc/00359/46988/

(c) 2016 Macmillan Publishers Limited, part of Springer Nature. All rights

reserved

\title{
Getting to the bottom of the ocean
}

De Lavergne Casimir ${ }^{1,{ }^{*}}$, Madec Gurvan ${ }^{1}$, Capet Xavier ${ }^{1}$, Maze Guillaume ${ }^{2}$, Roquet Fabien ${ }^{3}$

${ }^{1}$ Sorbonne Universités (Université Pierre et Marie Curie Paris 6)-CNRS-IRD-MNHN, LOCEAN Laboratory, F-75005 Paris, France

${ }_{2}^{2}$ Ifremer, Univ. Brest, CNRS, IRD, Laboratoire d'Océanographie Physique et Spatiale (LOPS), IUEM, F29280 Plouzané, France

${ }^{3}$ Department of Meteorology (MISU), Stockholm University, 10691 Stockholm, Sweden

* Corresponding author : Casimir de Lavergne, email address : casimir.delavergne@gmail.com 


\section{To the editor -}

The ocean is widely viewed as composed of an energetic surface layer in contact with the 20 atmosphere, overlying and interacting with a more quiescent and somewhat passive ocean interior. Available ocean observations and models mirror this view: sampling or resolution is highest near

22 the surface, rapidly decreases in the interior, and reaches lowest levels at abyssal depths (Fig. 1). Yet recent research suggests that the largely unchartered bottom boundary waters are as central to

24 ocean functioning as their surface counterparts ${ }^{1-7}$. Here, by summarizing identified key roles of the bottom boundary and highlighting persistent knowledge gaps, we overturn the common

26 surface-centric perception of the ocean and encourage new observational efforts to unveil and quantify bottom ocean phenomena. Without such efforts, we expect that bottom processes will

28 stand as a narrowing bottleneck in our understanding of the ocean's role in climate.

30 Accelerated by surface wind and thermohaline forcing or by tidal forces, oceanic flows rely largely on interactions with the slopes and roughness of the bottom topography for their ultimate

32 arrest $^{8,3-5}$. Though they set the energy and momentum balance of the ocean, these near-bottom dissipative processes remain rather poorly known, some of them still lacking identification or 34 understanding and most of them lacking accurate quantification ${ }^{8,9}$. The lack of a reliable closure of momentum and energy budgets hampers in turn our ability to describe and model the flow of

36 heat and other climatically important tracers across the oceans, both within deep and upper layers.

38 In particular, the concentration of energy dissipation along the bottom boundary is a key determinant of the large-scale distribution of ocean properties, and of the rate at which the

40 atmosphere and the deep ocean heat and carbon reservoirs communicate. The dissipation of oceanic flows is synonymous to a transfer of their kinetic energy to small-scale turbulence 42 through various instabilities. The resulting turbulent mixing redistributes seawater properties, balancing local transports by ocean currents and, more fundamentally, global sources and sinks

44 through the ocean's surface and bottom boundaries. The seafloor-catalysed energy dissipation is thus tied to elevated turbulent mixing rates, typically concentrated within the bottom few percents 
46 or 10-300 metres of the water column, that largely contribute to shape tracer distributions and to set the overall ventilation rate of the deep ocean.

Furthermore, the bottom enhancement of turbulence entails a near-bottom confinement of 50 mixing-induced density losses and of the associated upwelling that drains dense waters out of deep seas ${ }^{6,7}$. The along-topography upwelling is reinforced by geothermal heating, which further

52 lightens bottom-most waters, with global significance ${ }^{6}$. Because the injection of dense waters into deep basins occurs through downslope currents, both entry and exit routes of the abyss appear 54 confined to a thin bottom layer. Hence, in addition to hosting key boundary processes and exchanges, the bottom boundary layer stands out as the primary ventilation conduit of the abyssal 56 ocean.

58 However, bottom ocean waters also stand out as a major blind spot and critical chokepoint in our understanding and modelling of ocean heat and carbon storage and transports. Which boundary

60 processes and which dynamical regimes dominate the energy transfer to small-scale turbulence? How do they depend on topography scales and shapes? The possibility that submesoscale

62 currents, observed in the surface boundary layer and off steep continental slopes ${ }^{4,5,9}$, are also widespread along unstratified or rugged abyssal boundaries remains to be assessed. Overall, basic

64 knowledge of the thickness of the well-mixed bottom layer, of the near-bottom levels of stratification and mixing, and of the nature and rates of exchanges between the boundary layer

66 and the interior, together with their spatio-temporal variability, is lacking.

68 Improved process understanding may be achieved with high resolution idealized or regional model studies focusing on flow-topography interactions, instabilities and mixing ${ }^{3-5}$. But headway

70 will remain slow unless new in situ observations can bring into focus leading processes and provide a ground-truth reference. The thickness of the turbulent bottom boundary layer, and the

72 large depths and pressures found along most of the seabed (Fig. 1), pose challenging requirements on the nearness to topography and the depth sensors must reach. Ongoing

74 instrumental developments, including Deep Argo floats ${ }^{10}$, deep-sea gliders or terrain-following probes together with biochemical sensors, could rise to the challenge of mapping the ocean's 76 underside. In general, renewed attention to bottom dynamics and exchanges is imperative to uncover the key physical and biochemical phenomena that hide along the ocean floor.

\section{References}

1. Huussen, T. N., Naveira Garabato, A. C., Bryden, H. L. \& McDonagh, E. L. Is the deep Indian 84 Ocean MOC sustained by breaking internal waves? J. Geophys. Res., 117, C08024 (2012).

86 2. Polzin, K. L., Naveira Garabato, A. C., Abrahamsen, E. P., Jullion, L. \& Meredith, M. P. Boundary mixing in Orkney Passage outflow. J. Geophys. Res., 119 (2014). 
4. Molemaker, M. J., McWilliams, J. C. \& Dewar, W. K. Submesoscale instability and generation 94 of mesoscale anticyclones near a separation of the California Undercurrent. J. Phys. Oceanogr., 45, 613-629 (2015).

5. Gula, J., Molemaker, M. J. \& McWilliams J. C. Topographic generation of submesoscale

98 centrifugal instability and energy dissipation. Nat. Comm., 7, 12811 (2016).

100 6. de Lavergne, C., Madec, G., Le Sommer, J., Nurser, A. J. G. \& Naveira Garabato, A. C. On the consumption of Antarctic Bottom Water in the abyssal ocean. J. Phys. Oceanogr., 46, 635-661 102 (2016).

104 7. Ferrari, R., Mashayek, A., McDougall, T. J. \& Campin, J.-M. Turning ocean mixing upside down. J. Phys. Oceanogr., 46, 2239-2261 (2016).

106

8. Naveira Garabato, A. C. A perspective on the future of physical oceanography. Phil. Trans. $R$. 108 Soc. A., 370, 5480-5511 (2012).

110 9. McWilliams, J. C. Submesoscale currents in the ocean. Proc. R. Soc. A, 472, 20160117 (2016).

112 10. Zilberman, N. \& Maze, G. Report on the Deep Argo Implementation Workshop. Hobart May 5-7 ${ }^{\text {th }}$ 2015. http://archimer.ifremer.fr/doc/00281/39238/ (2015).

114

\section{Figure Legend}

118 Figure 1: Depth-distributions of seafloor area (black), ocean volume (blue), observational hydrographic sampling (orange), and number of model grid points (red), shown as a cumulative 120 percentage from the bottom upward. The gap to be bridged is well illustrated by the opposition between the depth-distributions of seafloor area and observational coverage or model resolution.

$12294 \%$ of hydrographic observations are concentrated in the upper 2,000 m, the depth range covered by autonomous Argo probes. New 'Deep Argo' probes, diving to 4,000 or 6,000 m, are

124 being developed to sample deeper waters ${ }^{10}$. Floats profiling to $4,000 \mathrm{~m}$ cover $88 \%$ of the ocean volume but only $47 \%$ of the ocean floor. Historical (1950-2014) observational sampling is 126 calculated from all temperature casts recorded in the most recent CORA database (http://doi.org/10.17882/46219). The state-of-the-art climate model grid taken as example is a 73-

128 level, nominally $1^{\circ} \times 1^{\circ}$ global ORCA mesh. 


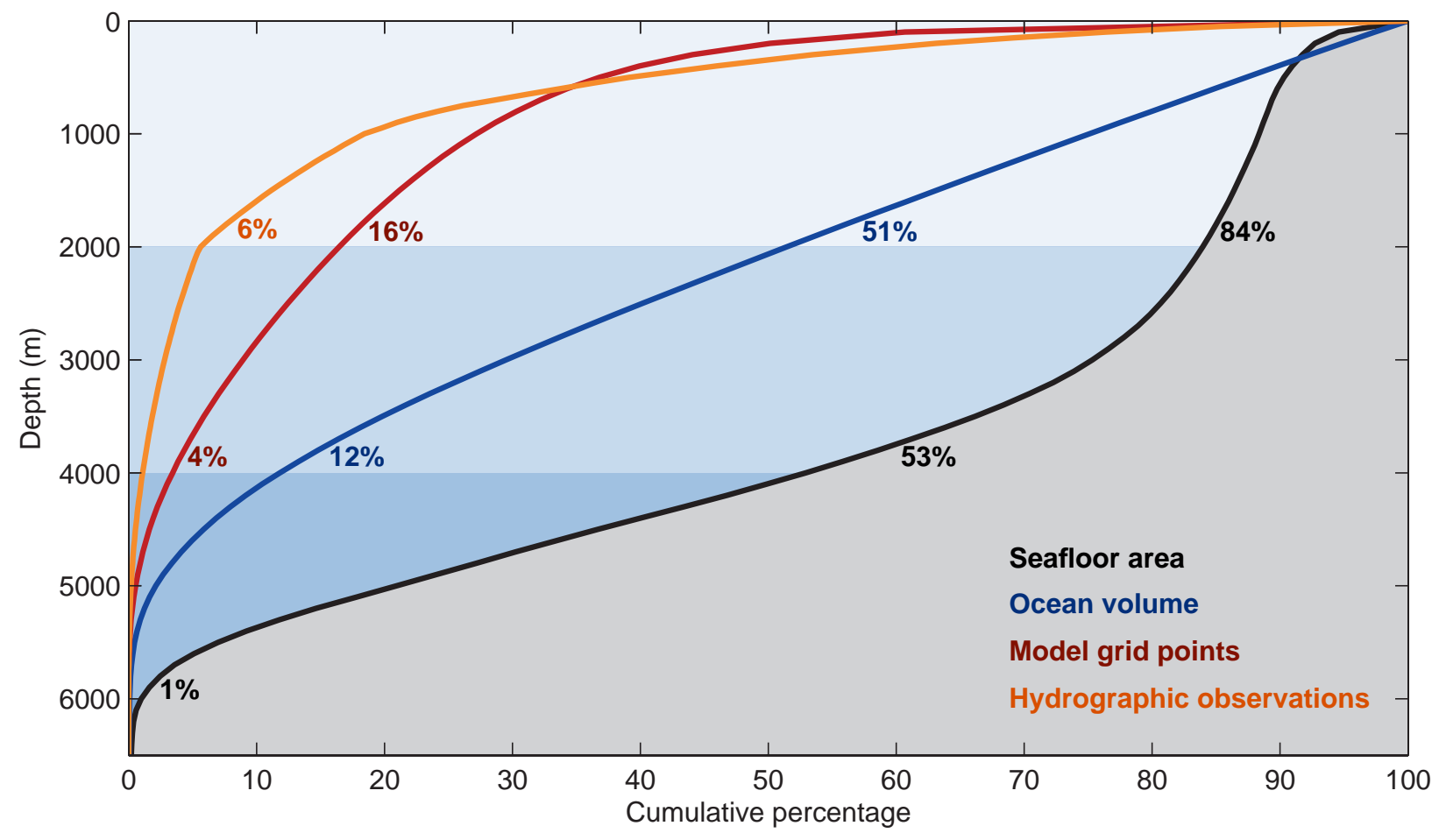

